\title{
Mitochondrial genome sequences from wild and cultivated barley (Hordeum vulgare)
}

\author{
Hiroshi Hisano ${ }^{1 \dagger}$, Mai Tsujimura ${ }^{2 \dagger}$, Hideya Yoshida ${ }^{1}$, Toru Terachi ${ }^{2}$ and Kazuhiro Sato ${ }^{1 *}$ (D)
}

\begin{abstract}
Background: Sequencing analysis of mitochondrial genomes is important for understanding the evolution and genome structures of various plant species. Barley is a self-pollinated diploid plant with seven chromosomes comprising a large haploid genome of $5.1 \mathrm{Gbp}$. Wild barley (Hordeum vulgare ssp. spontaneum) and cultivated barley (H. vulgare ssp. vulgare) have cross compatibility and closely related genomes, although a significant number of nucleotide polymorphisms have been reported between their genomes.

Results: We determined the complete nucleotide sequences of the mitochondrial genomes of wild and cultivated barley. Two independent circular maps of the 525,599 bp barley mitochondrial genome were constructed by de novo assembly of high-throughput sequencing reads of barley lines H602 and Haruna Nijo, with only three SNPs detected between haplotypes. These mitochondrial genomes contained 33 protein-coding genes, three ribosomal RNAs, 16 transfer RNAs, 188 new ORFs, six major repeat sequences and several types of transposable elements. Of the barley mitochondrial genome-encoded proteins, NAD6, NAD9 and RPS4 had unique structures among grass species.

Conclusions: The mitochondrial genome of barley was similar to those of other grass species in terms of gene content, but the configuration of the genes was highly differentiated from that of other grass species. Mitochondrial genome sequencing is essential for annotating the barley nuclear genome; our mitochondrial sequencing identified a significant number of fragmented mitochondrial sequences in the reported nuclear genome sequences. Little polymorphism was detected in the barley mitochondrial genome sequences, which should be explored further to elucidate the evolution of barley.
\end{abstract}

Keywords: Hordeum vulgare, Mitochondrial genome, De novo assembly, Comparative genomics

\section{Background}

Cultivated barley (Hordeum vulgare ssp. vulgare) was domesticated from its wild ancestral form $(H$. vulgare ssp. spontaneum) ca. 10,000 years ago [1, 2]. Cultivated and wild barley share closely related genomes that do not exhibit crossing or recombination barriers [3]. Barley is a self-pollinated diploid with seven pairs of chromosomes comprising a large haploid genome of $5.1 \mathrm{Gbp}$. The barley genome has been analyzed by BAC fingerprinting, BAC-end sequencing, whole-genome shotgun sequencing and transcriptome analysis [4]. The genome data also

\footnotetext{
* Correspondence: kazsato@rib.okayama-u.ac.jp

${ }^{\dagger}$ Equal contributors

${ }^{1}$ Institute of Plant Science and Resources, Okayama University, 2-20-1 Chuo,

Kurashiki, Okayama 710-0046, Japan

Full list of author information is available at the end of the article
}

include haplotype sequence information for several cultivated and wild barley lines, which provides 15 million SNPs among haplotypes.

The possibility of multiple domestication events for barley has been discussed based on phylogenic analyses of cloned genes $[2,5,6]$. The wild ancestral form of barley is widely distributed in the Near East to Central Asia, with a center in the Fertile Crescent. During the evolution of wild barley, its mitochondrial genomes might have diversified. For example, Ahokas [7] detected cytoplasmic male sterility, one of the main traits derived from the mitochondrial genome, in a wild barley germplasm. This finding indicates that the organellar genomes have differentiated in wild barley during its long evolutionary process. 
Although the gene contents of the mitochondrial genomes of flowering plants are nearly identical, gene order and genome configuration are highly variable even within a species due to genome rearrangement [8]. Tsunewaki et al. [9] explored the evolutionary dynamics of the wheat mitochondrial genome with respect to its structural differentiation during cereal evolution by comparing the nucleotide sequences of wheat genes to those of rice and maize.

The nuclear genome of barley is closely related to those of wheat and its relatives, i.e., members of the Triticeae tribe. The mitochondrial genome structure of common wheat (Triticum aestivum) cv. Chinese Spring is almost identical to that of the Chinese wheat cultivar Yumai, with differences of seven SNPs and 10 indels in non-coding regions [10, 11]. Noyszewski et al. [12] sequenced the Triticeae mitochondrial genomes of Aegilops longissima, T. turgidum and an alloplasmic line with the Ae. longissima cytoplasm that carries the T. turgidum nucleus, finding evidence suggesting that the alloplasmic condition accelerates evolution towards forming new mitochondrial genomes. However, to date, little information about the mitochondrial genome in barley has been published.

In a study investigating the chloroplast genome, which together with the mitochondrial genome composes the plasmon, Middleton et al. [13] found that the chloroplast sequences of cultivated and wild barley are closely related (sequence identity of $99.98 \%$ ). The divergence time of the two barley sequences was estimated to be $80,000 \pm 20,000$ years using semi-penalized likelihood. A comparison of the chloroplast genome sequences of cultivated barley and common wheat identified four deletions and five insertions greater than $50 \mathrm{bp}$ in the chloroplast genome sequence of common wheat. This similarity in chloroplast sequence indicates that cultivated and wild barley are more closely related to each other than cultivated barley and cultivated wheat.

The haplotypes used in the current study were the Japanese malting barley cultivar 'Haruna Nijo' and the wild barley accession 'H602'. Haruna Nijo has been extensively used as a crossing parent for developing high-quality malting barley cultivars in Japan. The origin of this cultivar can be traced to the haplotype of a malting cultivar introduced more than 100 years ago from Europe. Haruna Nijo has also been used to generate genomic resources, e.g., a BAC library [14], full-length cDNAs $[15,16]$, and whole genome shotgun sequence analysis [17]. Wild barley accession $\mathrm{H} 602$ has been used as a haplotype that is distantly related to Haruna Nijo and thus, a high-resolution transcript map of Haruna Nijo/H602 has been developed [18]. Both haplotypes may represent cultivated and wild barley gene pools, suggesting that they are appropriate materials for revealing the diversity of mitochondrial genomes in barley.
In this study, we determined the complete nucleotide sequences of the mitochondrial genomes of wild and cultivated barley and compared their structures. We then analyzed the gene contents and unique regions of each genome in detail at the sequence level. Since no mitochondrial genome sequence has been published for barley, we compared the resulting sequences to the published mitochondrial sequences of related species, i.e., wheat and its relatives, to clarify the evolutionary process of barley mitochondria.

\section{Methods}

\section{Plant materials and mitochondrial DNA extraction}

Mitochondrial DNA samples were extracted from etiolated seedlings barley cultivar 'Haruna Nijo' and wild barley accession 'H602' according to the method of Bonen and Gray [19], with minor modifications. Barley seeds were obtained from the National Bioresource Project of Barley, MEXT of Japan. Using discontinuous $(1.15,1.30$ and $1.45 \mathrm{M})$ sucrose density gradient centrifugation, DNase-I treated mitochondria were collected from the interface between 1.30 and $1.45 \mathrm{M}$ sucrose. The mitochondrial DNA samples were purified by $\mathrm{EtBr} / \mathrm{CsCl}$ density-gradient ultracentrifugation. For diversity analysis of SNPs, seed samples from 246 barley cultivars and four wild accessions preserved at the Institute of Plant Science and Resources, Okayama University, Japan were used.

\section{Sequencing, assembly and completion}

Both 454 sequencing with the GS-FLX Titanium platform (Roche Diagnostics K.K.), and Illumina sequencing with the MiSeq platform (Illumina, San Diego, CA, USA) were performed to generate longer reads by GS-FLX 454 system and correct read errors with high redundant shorter reads by MiSeq system. The libraries for 454 sequencing were prepared with GS Titanium Rapid Library Preparation Kit (Roche) and sequenced using GS FLX Titanium PicoTiterPlate Kit 70x75 (Roche) and GS FLX Titanium Sequencing Kit XL+ (Roche) following the manufacturer's instructions. GS FLX Titanium Rapid Library MID Adapter Kit (Roche), GS FLX Titanium LV emPCR Kit (Lib-L) v2 (Roche) and GS FLX Titanium emPCR Breaking Kit LV/MV 12pc (Roche) were used for adapter ligation and emulsion PCR during library and sequencing preparation. The libraries for Illumina sequencing were prepared with Nextera XT DNA Sample Preparation Kit (Illumina) and Nextera XT index Kit (Illumina) and sequenced with MiSeq Reagent Kit v2 500 cycles (Illumina) following the manufacturer's instructions.

The reads from 454 sequencing were assembled with Newbler 2.6 (Roche). The generated contigs were filtered by average depth $(>15)$. BLASTN analysis was performed to exclude contamination of chloroplast genome sequences. 
Filtered contigs were temporally ordered by hooking shared terminator reads in each contig.

Modified touchdown PCR [20] was performed to connect the contigs and to validate the SNPs with the following program: initial denaturation at $98{ }^{\circ} \mathrm{C}$ for 2 min, 10 cycles of a melting step at $98{ }^{\circ} \mathrm{C}$ for $10 \mathrm{~s}$, an annealing step at $65{ }^{\circ} \mathrm{C}$ decreased $1{ }^{\circ} \mathrm{C} /$ cycle for $15 \mathrm{~s}$ and an extension step at $72{ }^{\circ} \mathrm{C}$ for $1 \mathrm{~min}$, followed by 20 cycles of denaturation at $95{ }^{\circ} \mathrm{C}$ for $10 \mathrm{~s}$, annealing at $55{ }^{\circ} \mathrm{C}$ for $20 \mathrm{~s}$ and extension at $72{ }^{\circ} \mathrm{C}$ for $1 \mathrm{~min}$, with a final extension at $72{ }^{\circ} \mathrm{C}$ for $10 \mathrm{~min}$. The $10 \mu \mathrm{l}$ reaction mixture for PCR included $1 \mathrm{ng}$ mitochondrial DNA as template, $1 \times$ PrimeSTAR $^{\bullet}$ Max DNA Polymerase (TAKARA, Japan) and $0.5 \mu \mathrm{M}$ specific primers listed in Additional file 1: Tables S1a, b and Additional file 2: Tables S2. The PCR amplicons were checked by electrophoresis in $1.5 \%$ agarose gels (Wako, Japan) in TBE running buffer. Direct sequencing of the PCR amplicons was performed on a 3130xl Genetic Analyzer (Applied Biosystems, Waltham, MA, USA).

To increase the accuracy of genome sequencing, Illumina-reads from H602 and Haruna Nijo were mapped and aligned onto the reference mitochondrial draft genome of $\mathrm{H} 602$.

\section{Genome annotation and comparative analysis}

The genes encoding mitochondrial proteins and rRNAs were identified using BLASTN and MITOFY [21] based on the known annotation of the mitochondrial genes of wheat cv. Chinese Spring [10]. The tRNAscan-SE 1.21 server [22] was used to identify tRNAs. Repeat sequences ( $>100$ bp and $>80 \%$ identity) were identified by Align Sequences Nucleotide BLAST using mitochondrial sequences from Haruna Nijo. Partial sequences sharing more than $10 \%$ of nucleotides with the original genes were identified as pseudogenes. For transposable element analysis, CENSOR [23] was used with four sequence sources (database) including Poaceae, Oryza, Panicoideae and Triticum.

To identify novel ORFs, 'getorf' analysis in EMBOSS (http://emboss.sourceforge.net/apps/cvs/emboss/apps/ getorf.html) was conducted with the following parameters: predicted protein more than 100 aa in length; methionine as the start codon; circular DNA as the query sequence. After getorf analysis, the ORFs overlapping with known genes were eliminated.

\section{Alignments and phylogenetic analysis}

Phylogenetic analysis was carried out using concatenated nucleotide sequences of the 24 protein-coding genes (all electron transport chain genes and $c c m B, c c m C, c c m F C$, $c c m F N$, matR and $m t t B$ ) extracted from the complete mitochondrial genome sequences deposited in the public databases. All positions containing gaps and missing data were eliminated, yielding a total of 23,875 positions in the final dataset. Alignments and phylogenetic tree construction were performed by the maximum likelihood method with the MEGA5 program [24].

\section{SNP analysis}

A modified touchdown PCR procedure, as described previously, was performed for SNP analysis with specific primer pairs (Additional file 2: Table S2) using $50 \mathrm{ng}$ genomic DNA from cultivated and wild accessions of barley. PCR amplicons were sequenced with a 3130xl Genetic Analyzer.

\section{Results}

Sequencing and assembly of the mitochondrial genome in barley

The mitochondrial genomes of wild and cultivated barley were analyzed by 454 sequencing (Roche Diagnostics K.K.). A total of 954,203 and 256,123 reads corresponding to $280 \mathrm{Mbp}$ (average read length $586 \mathrm{bp}$ ) and 132 Mbp (514 bp) were generated from H602 and Haruna Nijo, respectively. These reads were independently de novo assembled using Newbler 2.6 (Roche) with average contig sizes of 993 and 1624 bp, N50 contig sizes of 915 and $1789 \mathrm{bp}$ and largest contig sizes of 76,676 and 56,491 bp for H602 and Haruna Nijo respectively. After trimming of duplicated sequences, these contigs were aligned in 18 contigs (525,551 bp) for $\mathrm{H} 602$ and 27 contigs $(525,569 \mathrm{bp})$ for Haruna Nijo (Additional file 3 : Tables S3a and S3b). The contig sequences were aligned, followed by sequencing of the PCR products with a 3130xl Genome Analyzer (see Additional file 1: Tables S1a and S1b for primer information). Draft master circular maps (master circles) of the mitochondrial genome were generated for both $\mathrm{H} 602$ and Haruna Nijo.

The Illumina MiSeq reads were mapped onto the master circles and re-aligned to develop high quality mitochondrial sequences. From the mitochondrial sequencing libraries of H602 and Haruna Nijo, 2,241,878 and $6,973,796$ paired reads were generated, respectively. Reads of H602 were trimmed with CLC genomic workbench 8.0 (Filgen Inc.), and the resulting pairs of 596,175 reads were mapped onto the draft master circle of H602. A 525,599 bp circular molecule with a G/C content of 44.2 \% (accession no. AP017300) was developed for H602. Using the same methods, another 525,599 bp circular molecule (accession no. AP017301) was developed for Haruna Nijo with pairs of 2,119,807 trimmed reads.

The alignment of two mitochondrial circular molecules identified three SNPs between H602 and Haruna Nijo at the positions of nt 510 (A/T, SNP1), 193,306 (G/T, SNP2) and 292,434 (A/C, SNP3). SNP1 was located in a genic region ( $\left.r p s 3 a \_p\right)$, and SNP2 and SNP3 were located in intergenic regions. These SNPs were validated by Sanger sequencing of PCR products, including 
the alleles amplified by specific primers (Additional file 2: Table S2).

\section{Annotation of the mitochondrial genome}

The master circles were annotated by BLAST analysis against the mitochondrial genome annotation databases MITOFY [21] and tRNAscan-SE 1.21 [22], and 33 protein-coding genes, three ribosomal RNAs and 16 transfer RNAs were identified (Fig. 1 and Additional file 4: Table S4). Of the 33 protein-coding genes, 18 encoded members of the electron transport chain and ATP synthase, including the following: nine subunits of complex I including nad1, nad2, nad3, nad4, nad4L, nad5, nad6, nad7 and nad9; an apocytochrome b subunit $(c o b)$ of complex III; three subunits of complex IV including $\operatorname{cox} 1, \operatorname{cox} 2$ and $\operatorname{cox} 3$; and five subunits of complex V including atp1, atp4, atp6, atp8 and atp9. Four additional protein-coding genes were involved in cytochrome c biogenesis, including $c c m B, c c m C, c c m F C$ and $c c m F N$. There were 10 genes for ribosomal proteins, namely rps1, rps 2 , rps3, rps4, rps7, rps12, rps13, rpl5, rpl16 and pseudogene $r p l 2 p$, as well as two other protein-coding genes, matR and $m t t B$. Among the protein-coding genes, there were two copies of nad3, nad9, ccmC, rps2 and rpl5. Among rRNA and tRNA genes, there were three copies of $r r m 5$, $\operatorname{rrn} 18, \operatorname{trn} D, \operatorname{trnfM} 2, \operatorname{trn} K$ and $\operatorname{trn} Q$ and two copies of rrn26 and $\operatorname{trn} R$. Of the two copies of $\operatorname{cox} 2 b$, the one nearest to $\operatorname{cox} 2 a$ was regarded as active $\operatorname{cox} 2 b$, whereas the other, which is located far from cox $2 a$, was regarded as a pseudogene due to the general rule of cis-splicing between $\operatorname{cox} 2 a$ and $\operatorname{cox} 2 b$.

\section{Prediction of new genes}

We used the 'getorf' command in EMBOS to identify unique genes in the barley mitochondrial genome, leading to the prediction of 188 new ORFs ( $>100$ amino acids). After excluding any partially overlapped ORFs with known genes in the same translational frame, 142 ORFs were identified as novel genes (Additional file 5: Table S5). The total length of these novel ORFs was $66,180 \mathrm{bp}$, which occupied $12.6 \%$ of the barley mitochondrial genome.

\section{Identification of transposable fragments}

CENSOR [23] was used to identify integrated transposable elements showing similarities to the sequences in the Poaceae (grass family), Oryza (rice), Panicoideae (maize, sugarcane, sorghum and millet) and Triticum (wheat, barley and rye) databases. A total of 88 (15,202 bp total) DNA transposons, LTR retrotransposons and non-LTR

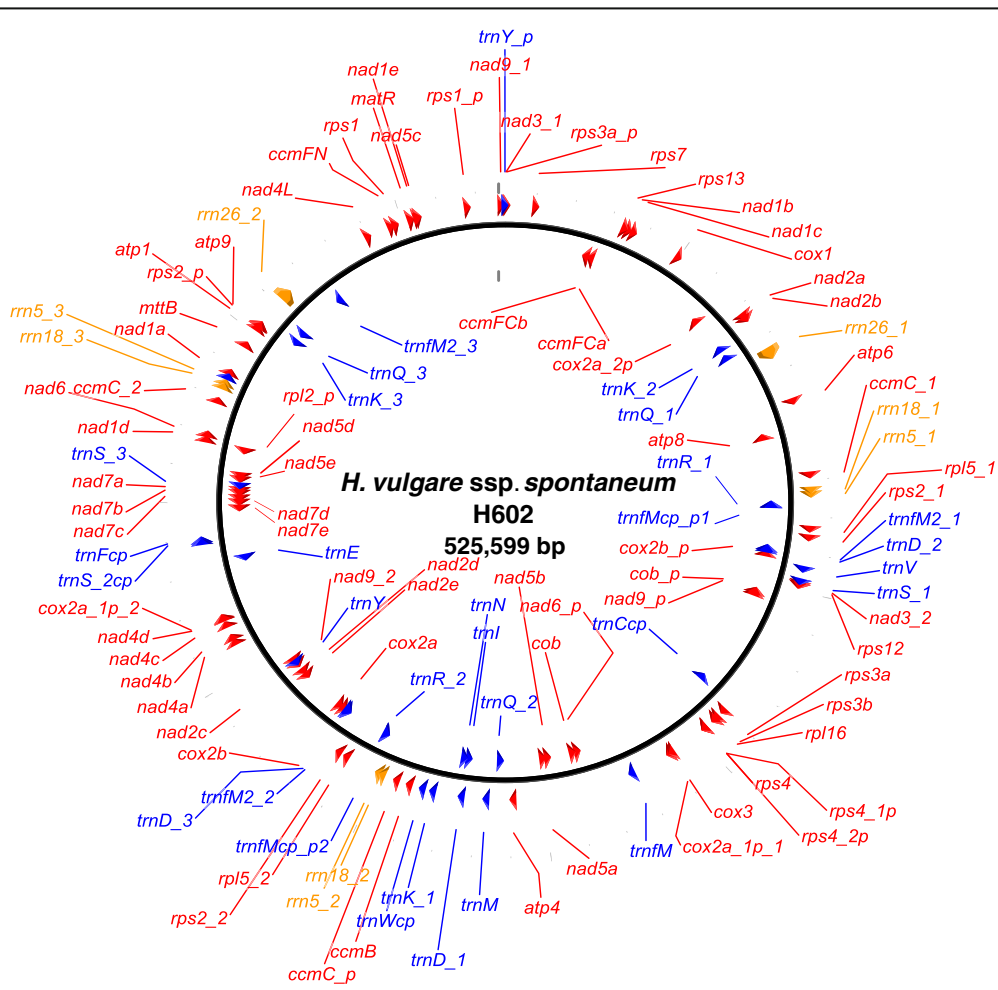

Fig. 1 Positions of genes on the barley mitochondrial genome map (Hordeum vulgare ssp. spontaneum acc. H602). Genes encoding proteins (red), rRNA (orange) and tRNA (blue) are shown outside (forward direction) and inside (reverse complementary direction) the circle. Arrowhead indicates gene or exon, $p$ indicates pseudogene, $a, b, c, d$ and $e$ indicate cis- and trans-spliced genic exon and underscores followed by numbers indicate gene copies 
retrotransposons were found in Poaceae, 59 (9586 bp) were found in Oryza and 73 (2682 bp) were found in Panicoideae (Table 1). By contrast, only 16 LTR retrotransposon and two non-LTR retrotransposons (cumulatively $2246 \mathrm{bp}$ ) were found in Triticum.

\section{Repeat sequences and chloroplast-derived region in the mitochondrial genome}

Six major repeat sequences (>1000 bp, identity $100 \%$ ) and 17 repeat sequences $(>100 \mathrm{bp}$, identity $>99 \%)$ were found in the barley mitochondrial genome (Fig. 2 and Additional file 6: Table S6). There were four repeats in No. 18 and three in Nos. 6, 12, 15, 17 and 21. The largest repeat was $25,303 \mathrm{bp}$, corresponding to the contig_H602_0022-0039-0169-0014 (Additional file 3: Tables S3a and S3b).

According to previous reports [25, 26], several tRNAs that function in the mitochondria originated in the chloroplast. In the barley mitochondrial genome, $\operatorname{trn} C$, $\operatorname{trn} F$, trnS2 and $\operatorname{trn} W$ could represent tRNA genes of chloroplast origin (Additional file 4: Table S4). We analyzed the sequence similarity between the mitochondrial genome of $\mathrm{H602}$ and the chloroplast genomes [13] by BLASTN analysis of barley cvs. Barke (KC912687) and Morex (EF115541) and found three homologous regions (>1 kbp) (Additional file 7: Table S7). Homologous region 1, harboring trnfM, trnR, rps14 and psaA, contained $6307 \mathrm{bp}$ in the $\mathrm{R} 1$ repeat region (Additional file 6: Table S6), suggesting that the mitochondrial genome contained two copies of homologous region 1. Among these genes, trnfM and $\operatorname{trnR}$ were identified using the mitochondrial genome annotation database, whereas rps14 and psaA were not identified. Homologous region 2 was $2265 \mathrm{bp}$ in length and harbored $\operatorname{trn} V$. Homologous region 3 contained $1110 \mathrm{bp}$ of sequences specific to the chloroplast sequence of Barke. Although homologous regions 1 and 2 were found in the chloroplast genomes of several monocot species such as rice and maize, region 3 was only found in Barke. Fifteen other homologous regions, ranging from 29 to $558 \mathrm{bp}$ in length, were also found by BLASTN analysis (Additional file 7: Table S7).

\section{Transfer of mitochondrial DNA into the barley nuclear genome}

We analyzed nucleotide sequence similarities between the mitochondrial genome of H602 and the published barley genome sequences (Hordeum_vulgare.ASM32608v1.31.dna_sm.genome.fa.gz), comprising pseudomolecules of seven chromosomes and un-anchored scaffolds and contigs published in Ensembl Plants Hordeum vulgare (http://plants.ensembl.org/Hordeum_vulgare/Info/Index), using BLASTN analysis (Additional file 8: Table S8). Using the threshold $\mathrm{E}$-value $=0$, aligned sequence length $>400 \mathrm{bp}$, we detected 621 barley genome sequences showing similarities to the barley mitochondrial genome sequence. Of these, many of the longest un-anchored genome sequences appeared to be mitochondrial sequences, since the lengths of un-anchored sequences and aligned sequences were quite similar. For example, the sequence of morex_contig_70567, which was 23,224 bp in length, aligned completely with the mitochondrial sequence without mismatches or gaps. Of the genome sequences within the threshold, seven were derived from the chloroplast genome and 93 were un-anchored contigs that were

Table 1 Transposable elements in the barley mitochondrial genome

\begin{tabular}{|c|c|c|c|c|c|c|c|c|}
\hline \multirow[b]{2}{*}{ Repeat class } & \multicolumn{2}{|l|}{ Poaceae $^{a}$} & \multicolumn{2}{|l|}{ Oryza $^{a}$} & \multicolumn{2}{|l|}{ Panicoideae $^{a}$} & \multicolumn{2}{|l|}{ Triticum $^{a}$} \\
\hline & No. of fragments & Length & No. of fragments & Length & No. of fragments & Length & No. of fragments & Length \\
\hline DNA transposon & 13 & 837 & 9 & 522 & 8 & 406 & - & - \\
\hline EnSpm & 1 & 58 & 1 & 97 & 2 & 93 & - & - \\
\hline Harbinger & 1 & 38 & 1 & 38 & - & - & - & - \\
\hline Helitron & 5 & 428 & 2 & 157 & - & - & - & - \\
\hline MuDR & 5 & 277 & - & - & 5 & 277 & - & - \\
\hline hAT & - & - & 4 & 194 & - & - & - & - \\
\hline Other & 1 & 36 & 1 & 36 & 1 & 36 & - & - \\
\hline LTR retrotransposon & 57 & 10,210 & 41 & 572 & 49 & 8591 & 16 & 2043 \\
\hline Copia & 19 & 3574 & 10 & 1947 & 14 & 2306 & 7 & 890 \\
\hline Gypsy & 38 & 6636 & 31 & 6139 & 35 & 6285 & 9 & 1153 \\
\hline Non-LTR & 18 & 4155 & 9 & 978 & 16 & 3685 & 2 & 103 \\
\hline L1 & 17 & 3911 & 6 & 572 & 16 & 3685 & 1 & 41 \\
\hline Other & 1 & 244 & 3 & 406 & - & - & 1 & 62 \\
\hline Total & 88 & 15,202 & 59 & 9586 & 73 & 12,682 & 18 & 2146 \\
\hline
\end{tabular}

${ }^{\mathrm{a}}$ Sequence sources (database) for transposable element analysis in CENSOR 


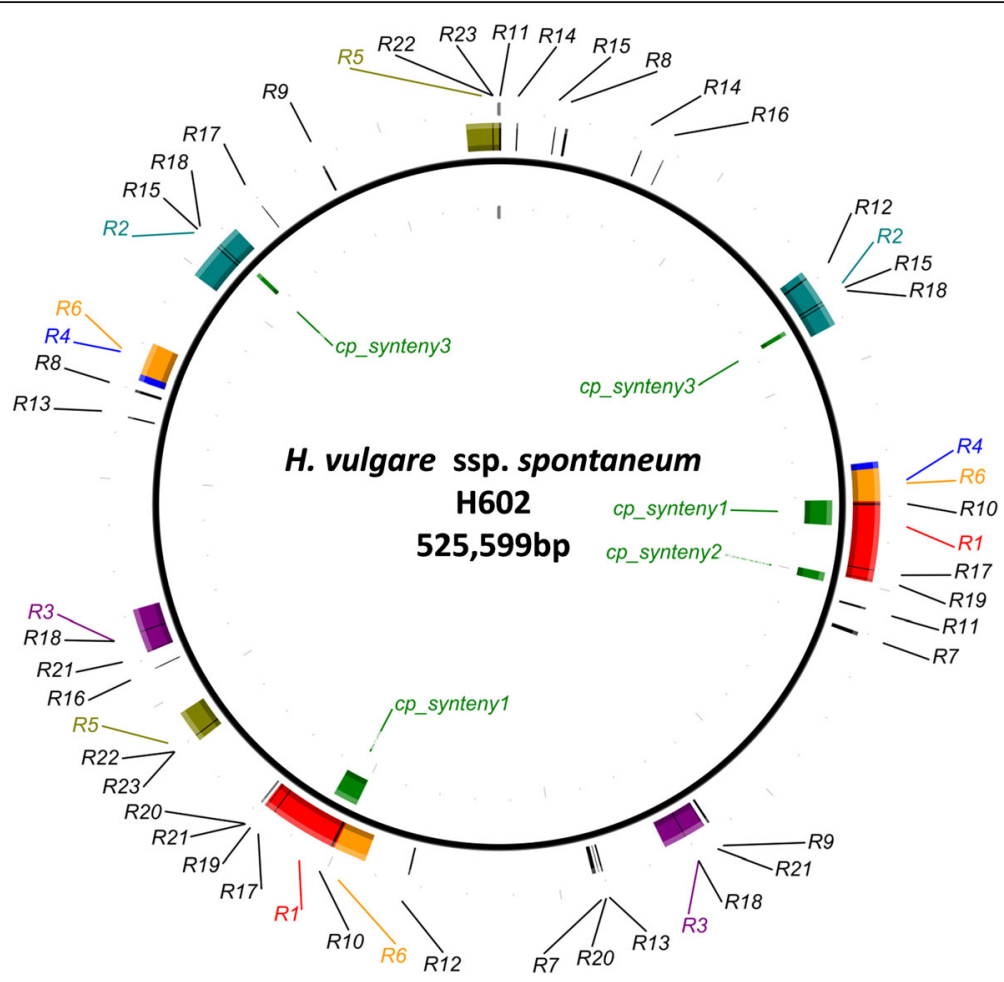

Fig. 2 Repeat sequences and integrated chloroplast genome in the map of the barley mitochondrial genome of accession H602. Repeat sequences in different colors are mapped onto the barley mitochondrial genome (outside). Detailed information about these repeats is shown in Additional file 6: Table S6. The predicted sequences integrated from the chloroplast genome are shown inside the circle

potentially derived from the mitochondrial genome in the published barley genome sequence obtained by wholegenome shotgun sequencing. Apart from the misidentified mitochondrial sequence, nuclear sequences of mitochondrial origin (Nuclear mitochondrial DNA = NUMTs) were observed. Of the 561 possible NUMTs anchored to pseudomolecules, $11,579 \mathrm{bp}$ of sequence on chromosome $6 \mathrm{H}$ was the longest that aligned with the mitochondrial genome. The total amounts of aligned sequences varied among chromosomes, ranging from $16,427 \mathrm{bp}(5 \mathrm{H})$ to $150,387 \mathrm{bp}$ $(3 \mathrm{H})$, indicating that the mitochondrial sequences were not evenly transferred to the nuclear genome [27].

\section{SNP analysis of barley germplasm}

Barley germplasm comprising 246 cultivars from 40 regions (countries) and four wild accessions were genotyped at three SNPs that were identified between the H602 and Haruna Nijo mitochondrial genomes. Of the 188 cultivars showing a nucleotide call on SNP1 (H602/ Haruna Nijo: A/T), 13 and 175 contained an A and T, respectively (Additional file 9: Table S9). Of the four wild barley accessions, two contained $\mathrm{A}$ and two contained $\mathrm{T}$. For SNP2 (T/G), all of the cultivars and three wild barley accessions contained G, but one wild barley, H627, contained T. For SNP3 (C/A), 184 of 223 cultivars and one wild barley (H685) contained A (Additional file 9:
Table S9). Most Ethiopian cultivars contained C in SNP3, although other regional differences in the allele distribution were not obvious.

Comparative genomics of barley mitochondria with those of wheat and other monocot species

We compared the genes/proteins in the barley mitochondrial genome with those in other grass species including common wheat ( $T$. aestivum) and wheat ancestors $(T$. timopheevii and Ae. speltoides), perennial ryegrass ( $L$. perenne), sorghum (S. bicolor), maize ( $Z$. mays) and rice (O. sativa). Barley nad6 encoded NAD6, a 312 aa polypeptide (Table 2, Fig. 3 and Additional file 10: Figure S1). The sequence comprising aa 1 to 215 in NAD6 was conserved among grass species, whereas the C-terminus is highly variable. Barley nad 9 was conserved among grass species except wheat, which contained a longer nad9 due to frame-shifting caused by a $4 \mathrm{nt}$ insertion/deletion (Table 2, Fig. 3b and Additional file 10: Figure S1). The rps4 gene had 39-nt imperfect repeats corresponding to 13 aa (Fig. 3c and Additional file 10: Figure S1). Barley rps 4 had three repeats, whereas wheat has one and rice, maize and perennial ryegrass each have partial one (Fig. 3c). The genes cox1, atp6, rps2, rpl5 and rrn18 also varied between barley and wheat (Table 2). 
Table 2 Differences in mitochondrial genes encoding proteins between barley and wheat

\begin{tabular}{|c|c|}
\hline $\begin{array}{l}\text { Gene } \\
\text { in barley }\end{array}$ & Differences from wheat \\
\hline \multirow[t]{2}{*}{ nad6 } & 1. Low homology at 3' side (from 646 to 738 nt). \\
\hline & $\begin{array}{l}\text { 2. Additional } 201 \mathrm{nt} \text { on } 3^{\prime} \text { side ( } 67 \text { amino acid on the C- } \\
\text { terminus) because of stop codon substituted to leucine } \\
\text { caused by A to T substitution at } 737 \mathrm{nt} \text {. }\end{array}$ \\
\hline nad9 & $\begin{array}{l}\text { Deficient of } 97 \text { aa on } 5^{\prime} \text { side, however identical to ones } \\
\text { in rice, bamboo, maize, sorghum and ryegrass. }\end{array}$ \\
\hline \multirow[t]{2}{*}{$\operatorname{cox} 1$} & Two additional C-terminal aa. \\
\hline & Wheat cox 1 is the same as that of rice and bamboo. \\
\hline atp6 & Low homology at $5^{\prime}$ side $e^{a}$. \\
\hline rps2 & Low homology at $3^{\prime}$ side ${ }^{b}$. \\
\hline \multirow[t]{2}{*}{ rps4 } & $\begin{array}{l}\text { Insertion of } 78 \text { nt ( } 26 \text { aa) caused by multiple repeat } \\
\text { sequences. }\end{array}$ \\
\hline & $\begin{array}{l}\text { Barley has three of } 39 \mathrm{nt} \text { repeat sequence, whereas wheat } \\
\text { has one of this sequence. }\end{array}$ \\
\hline \multirow[t]{2}{*}{$r p / 5$} & Difference of 10 aa on C-terminus. \\
\hline & Wheat $r p / 5$ is same as that of rice, bamboo and ryegrass. \\
\hline$r r n 18$ & Insertions of $18 \mathrm{nt}$ and $4 \mathrm{nt}$. \\
\hline
\end{tabular}

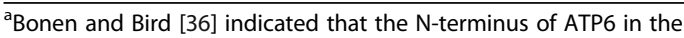
mitochondrial genome had interspecies variation caused by processing ${ }^{b}$ Kubo et al. [37] reported RPS2 in barley (BAD61010) and interspecies variation in Triticeae at its $\mathrm{C}$-terminus

We analyzed the evolutionary relationships among grass species by examining the DNA sequences of proteincoding genes in the mitochondrial genome. A phylogenetic tree constructed in MEGA5 is shown in Fig. 4. Barley belonged to the Triticeae clade, which included T. aestivum, T. timopheevii and Ae. speltoides. L. perenne was placed closer to barley than to S. bicolor, Z. mays and $O$. sativa, which belonged to another clade.

\section{Discussion}

\section{Features of the barley mitochondrial genome}

In this study, we determined the complete nucleotide sequences of mitochondrial genomes from the wild barley accession H602 and the Japanese malting cultivar Haruna Nijo. These two haplotypes differ in origin and have sufficient polymorphisms in the nuclear DNA sequences for a proper comparison [28]. However, we detected a high level of sequence identity (only three SNPs in 525,599 bp) between the two mitochondrial genomes; the mitochondrial genomes of these wild and cultivated barley lines were almost identical in terms of both nucleotide sequence and genome structure.

Our annotation indicated that the barley mitochondrial genome contains a total of 96 genes $(51,476 \mathrm{bp}$, excluding pseudogenes; Additional file 4: Table S4). These genes share similar components with genes in the wheat mitochondrial genome [10], as described in the Results and
Additional file 4: Table S4. ORF prediction also suggested that the genome contains 142 ORFs $(66,180 \mathrm{bp}$ in total) comprising $16 \%$ of the total genome (Additional file 5: Table S5). Six types of repeated sequences (R1 to R6) larger than $1 \mathrm{kbp}$ were found in the barley mitochondrial genome (Additional file 6: Table S6). The repeated sequences were $100 \%$ identical to each other and are located in direct orientation. There were two copies of R1 to R5 and three copies of R6 (Additional file 6: Table S6). Using CENSOR analysis, we identified a series of transposon types (Table 1). The total sequence lengths of Copia, Gypsy and non-LTR retrotransposons in the mitochondrial genome were 3574, 6636 and 4155 bp, respectively. In the nuclear genome of barley (63 Mbp BAC end sequences, [4]), ca. $81.80 \%$ is occupied by mobile elements, with a retroelement/DNA transposon ratio of $12.16 \%$. Of these, the ratios of Copia, Gypsy and non-LTR retrotransposons are 13.66, 20.84 and $0.31 \%$, respectively. We found the retroelement/DNA transposon ratio in the mitochondrial genome to be $17.16 \%$, which is much higher than that of the nuclear genome. The barley mitochondrial genome appears to have more Gypsy elements and many more nonLTR retrotransposons than the nuclear genome.

The barley mitochondrial genome contained three chloroplast-homologous sequences (6307, 2265 and 11 $10 \mathrm{bp}$ ) larger than $1 \mathrm{kbp}$ (Additional file 7: Table S7). Of these, two larger sequences were homologous to the chloroplast sequences of five barley cultivars [13] and even to those in wheat and rye. This result indicates that these homologous sequences were transferred from the chloroplast genome to the mitochondrial genome before the divergence of barley from a wheat/rye lineage. Another region $(1110 \mathrm{bp})$ showed sequence similarity only to the chloroplast genome of barley cv. Barke. This 1110 bp sequence could be cultivar specific, and its transfer from the mitochondrial genome to the chloroplast genome might have occurred quite recently only in this cultivar. However, gene transfer between genomes is usually restricted to transfer from the chloroplast and mitochondria to the nucleus, known as nupt and numt, respectively, and from the chloroplast to mitochondria. It is highly improbable that we encountered a rare transfer event from the mitochondria to chloroplasts in this cultivar. It is likely, therefore, that this $1110 \mathrm{bp}$ mitochondrial sequence was misassembled with the chloroplast genome of cv. Barke. The chloroplast-homologous sequence of $6307 \mathrm{bp}$ in the barley mitochondrial genome was longer than the corresponding sequence in the wheat mitochondrial genome, and annotation information (Additional file 4: Table S4) revealed that the tRNAs trnR and trnfM were included in this fragment. In addition, the 2265 bp sequence contained trn $V$. Perhaps these chloroplast-derived tRNAs function in the barley mitochondria, although experimental evidence is needed to support this notion. 


\section{a}

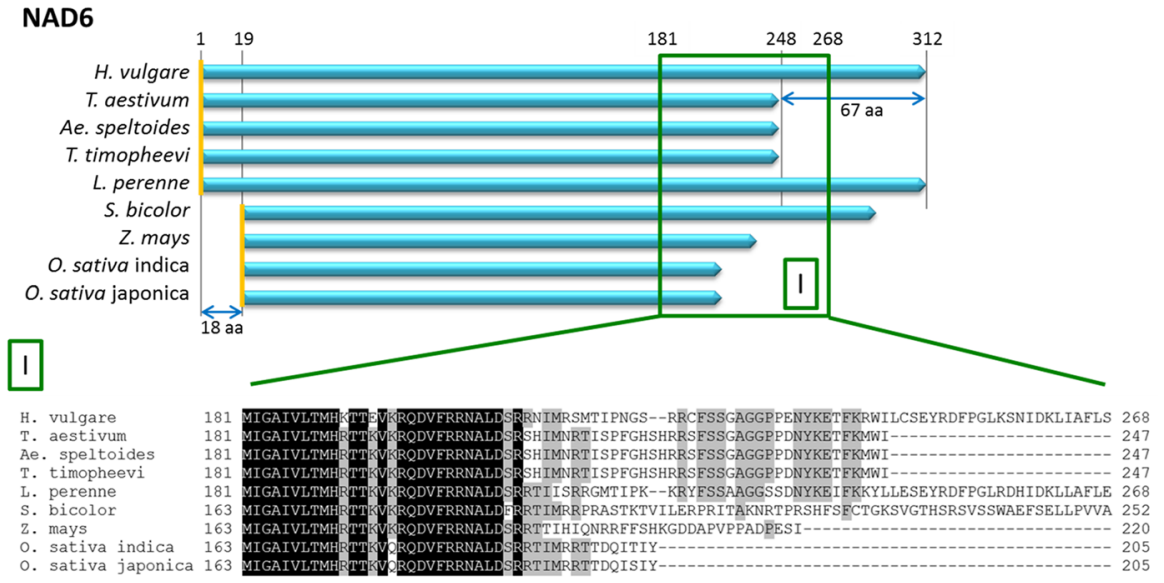

b

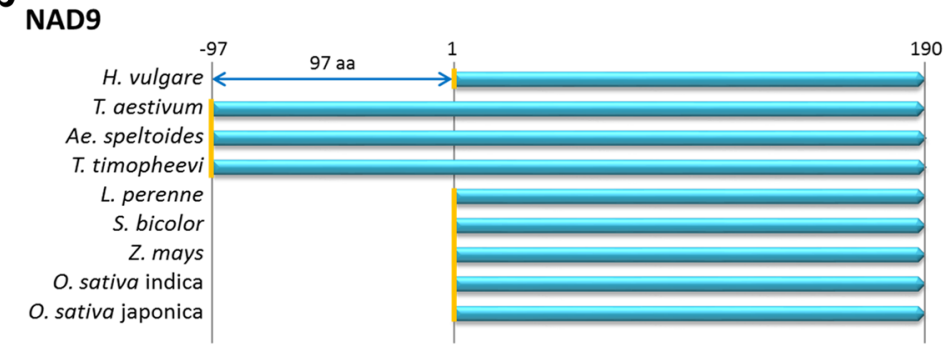

C

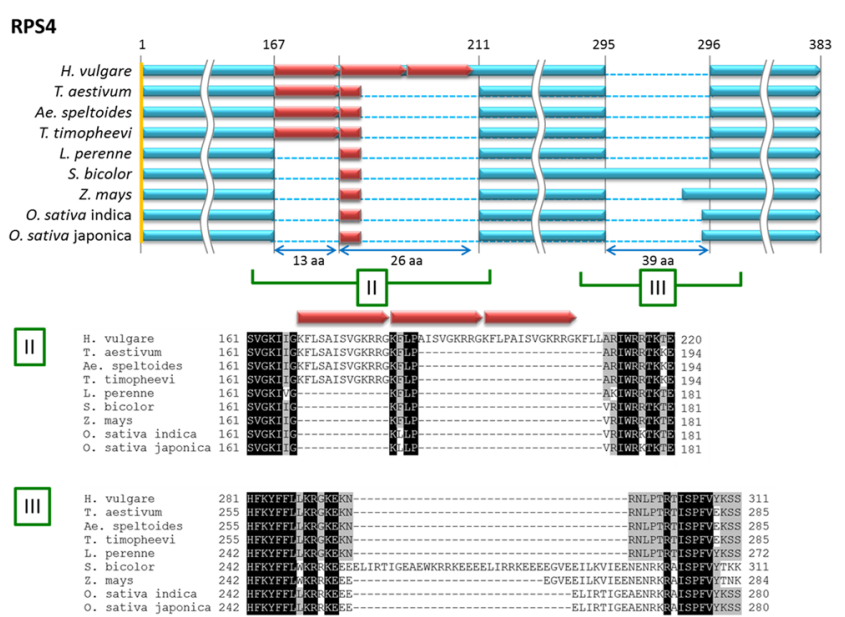

Fig. 3 Structures of the encoded protein sequences in various grass species. The structures of a NAD6, b NAD9 and c RPS4 in the barley mitochondrial genome were compared with those of seven other grass species and subspecies (T. timopheevii, Ae. speltoides, L. perenne, S. bicolor, Z. mays, O. sativa ssp. japonica and O. sativa ssp. indica). Light blue bars show the primary structures of proteins, and orange lines indicate the start codon. The numbers above the light blue bars indicate the position from the start codons of barley proteins. a Green box I indicates the non-conserved amino acid sequences in NAD6 among grass species. b NAD9 of T. aestivum, Ae. speltoides and T. timopheevii have an additional 97 aa on their N-terminal side compared with those of other grass species including barley. c Red bars indicate 13 aa repeats. Green boxes I/ and III indicate species-specific amino acid sequences in RPS4

\section{Diversity of barley mitochondrial sequences}

In the present study, only a few nucleotide polymorphisms were found between wild barley and cultivated barley mitochondrial haplotypes. Barley cultivation has a history of ca. 10,000 years after domesticated from its wild progenitor. According to the estimation of domestication mutation events on the Btr1 (brittle rachis 1) and Btr2 loci, which are responsible for the reproductive barrier via seed dispersal [2], there are two main sources of cultivated barley ancestors. It is surprising that the organellar genome of the wild haplotype used in this study was quite similar to that of the cultivated barley Haruna Nijo. 
Of the SNPs detected in four wild and 246 cultivated barleys, only one wild barley showed an identical haplotype to H602 that was not found in the cultivated barleys examined (Additional file 9: Table S9). Interestingly, the haplotype in Haruna Nijo was also detected in wild barley accession H685. Among the three SNPs, the H602 allele of SNP3 was abundant in North African and Ethiopian landraces, indicating that SNP3 can be traced to the germplasm distributed in North Africa, with an origin related to wild barley accession H695. The haplotype with the H602 alleles in SNP1 and SNP3 was also present in both wild and cultivated barley. Since there is no crossing barrier between the wild ancestral form and cultivated barley, it is possible that the mitochondrial genome of $\mathrm{H602}$ resulted from hybridization between wild and cultivated barley. More extensive sequencing of the mitochondrial genome is needed to elucidate mitochondrial genome evolution in wild and cultivated barley.

\section{Genome integration}

Wicker et al. [29] reported that the mitochondrial genome was included in the nuclear genome, as revealed by lowredundancy shotgun genome sequencing. According to BLASTN sequence similarity analysis (threshold: $\mathrm{E}=0$, alignment length $>400 \mathrm{bp}$ ) in the present study, many of the published genome sequences shared similarity with the mitochondrial genome (Additional file 8: Table S8). The length of total integration of the mitochondrial genome into the chromosome-anchored nuclear genome sequence was $595,866 \mathrm{bp}$, which is estimated to represent $0.01 \%$ of the total nuclear genome size in barley (5.1 Gbp). However, these mitochondrial sequences were not distributed evenly on the chromosomes, indicating that there are hot spots of chromosomal regions where mitochondrial sequences had transferred (Additional file 8: Table S8). The mitochondrial genome shared similarity with many of the long un-anchored sequences, which could represent sequences corresponding to the mitochondrial genome itself generated from wholegenome shotgun sequencing. The results also suggested that the plastid genome shares 18,215 bp of sequence with the mitochondrial genome, which had three duplicated regions corresponding to $7.54 \%(10,240 \mathrm{bp})$ of the total plastid genome (135,802 bp). Thus, the current complete barley mitochondrial genome sequence represents an important tool for annotating the nuclear genome sequence, as well as the mitochondrial sequence itself spuriously included in the previously reported nuclear genome sequence.

\section{Comparative genomics with other crops}

The size of the barley mitochondrial genome was 16 and $7 \%$ larger than that of wheat $(452,528 \mathrm{bp}$ for $\mathrm{cv}$. Chinese Spring) and rice (490,520 bp for cv. Nipponbare), respectively, but $30 \%$ smaller than that of perennial ryegrass $(678,580 \mathrm{bp})$. The barley mitochondrial genome was comparable in size to those of maize $(535,825$ and 557,162 bp for CMS-T and CMS-S type, respectively, and 569,630 bp for NB type from the inbred line B37N). Multiple genome alignment analysis using progressiveMauve [30] revealed the presence of more than 70 short syntenic regions between barley and wheat (data not shown). This complex pattern of fragmented synteny prevented us from reconstructing the evolutionary process by

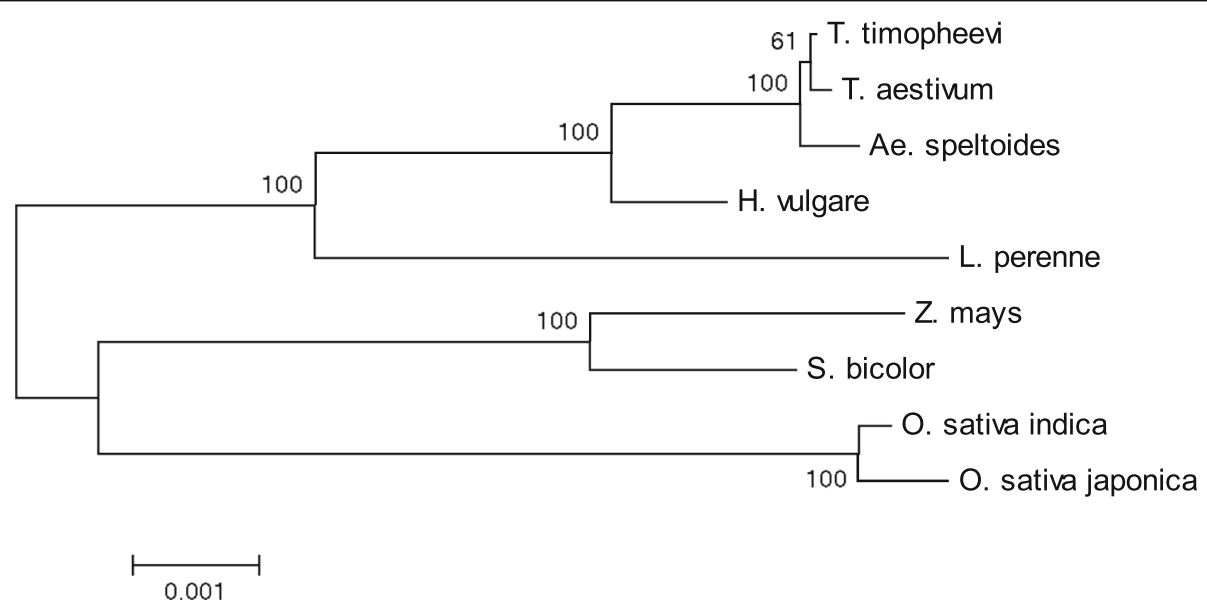

Fig. 4 Phylogenetic tree constructed using the DNA sequences of 24 protein-coding genes comprising a total of 23,875 bp in the mitochondrial genomes of eight grass species and subspecies by the maximum likelihood method. The protein-coding genes include all electron transport chain genes, $c \mathrm{cmB}, \mathrm{ccmC}, \mathrm{ccmFN}$, matR and $m t t B$. The sequences of these genes can be found under the following accession numbers: AP013106 (T. timopheevii), AP013107 (Ae. speltoides), JX999996 (L. perenne), DQ984518 (S. bicolor), AY506529 (Z. mays), BA000029 (O. sativa ssp. japonica) and DQ167399 (O. sativa ssp. indica). The percentage of trees in which the associated taxa clustered together in 1000 replicates of the bootstrap test is shown next to the branches. Scale bar indicates the number of base substitutions per site 
which extensive rearrangements occurred between the two mitochondrial genomes.

By contrast, a high proportion of genes in the mitochondrial genome of barley were almost the same as those of other grass species. In particular, the nucleotide sequences of protein-coding genes of the electron transport chain were highly conserved among grasses, although the presence of a few variations allowed us to construct a phylogenetic tree showing clear differentiation between Triticeae and other grass species (Table 2 and Fig. 3). Figure 4 shows a phylogenetic tree reconstructed from the nucleotide sequences of protein coding genes in the electron transport chain and those that function in cytochrome $\mathrm{c}$ biogenesis, as well as matR and $m t t B$. The tree indicates that barley is relatively closely related to wheat, as expected. The analysis using the program MITOFY also shows the sequences of individual genes of barely are closely related to those of corresponding genes of wheat. Therefore, there is no indication that any genes of barely have derived from other unrelated plant species by horizontal gene transfer.

Based on the analysis of the sequences of nuclear genes encoding multi-domain plastid acetyl-CoA carboxylase (ACCase) and plastid 3-phosphoglycerate kinase (PGK), it was estimated that Hordeum diverged from the Triticum/ Aegilops lineage ca. 11 million years ago (MYA) [31]. On the other hand, comparative chloroplast genome analysis led to the estimate that barley diverged from rye and wheat approximately 8-9 MYA [13]. Since barley shares a homoeologous chromosome system with wheat, only translocations differentiate the barley and wheat genomes.

Although the gene contents in the barley mitochondrial genome were quite similar to those of wheat [10], three genes (nad6, nad9 and rps4) differed considerably between barley and wheat in terms of structure (Table 2, Fig. 3 and Additional file 10: Figure S1). Compared to wheat nad6, barley nad6 contained a 198 nt extension in the 3 ' coding region. This extension may produce a NAD6 polypeptide that is 66 aa longer in barley than in wheat. Although the length of NAD6 deduced from the genome sequence of perennial ryegrass was nearly identical to that of barley, significant variations in both length and sequence were observed in the C-terminal half of NAD6 among grass species (Fig. 3a). NAD6 is a hydrophobic membrane protein and a component of the NADH dehydrogenase complex (complex I). Complex I consists of approximately 50 proteins, seven subunits of which are encoded by the mitochondrial genome [32]; it is difficult to imagine that NAD6 variants are properly integrated into such a sophisticated molecular machinery. To investigate the possibility that all grasses actually contain a short conserved NAD6, we looked for a potential stop codon before an in-frame stop codon in each nad6 gene that might have been created by RNA editing. Sequence comparison, however, showed no clear indication that a new stop codon was created and that premature termination helped increase the similarity among NAD6 polypeptides. It should be noted, however, that nad6 could be expressed from mRNAs lacking a stop codon in Arabidopsis [33]. In Arabidopsis (and cauliflower), nad6 mRNA was processed upstream of the in-frame stop codons, and the resulting mRNAs lacking stop codons were translatable [33]. This observation suggests that mRNA processing occurs at some points before an inframe stop codon and that processing cancels apparent length variations in nad6 genes among grass species. If this occurs, highly similar NAD6 polypeptides will be translated and integrated into complex I of each grass species. Determining the amino acid sequence constituting the C-termini of NAD6 from barley and other grasses will help test this notion.

The nad9 gene from barley shared identical sequences with those of most grass species. An exception was wheat nad9, which is 291 nt longer than nad9 genes from barley and other species. Two possible initiation codons (ATG) are present in the $5^{\prime}$ region of wheat nad9, and if the second ATG is used for translation, as suggested by Lamattina et al. [34], a similar polypeptide to that of barley will be produced in wheat.

The rps 4 gene contains variable numbers of a 39 nt repeat, depending on the species; this repeat is categorized as a minisatellite. Some plant mtDNAs contain minisatellites, most of which are located in intergenic regions, although a few occur in gene-coding and intronic regions [35]. The size of the abovementioned minisatellite (39 nt) is a multiple of three, which may explain why this minisatellite has survived in the coding region of rps 4 even after the divergence of the barley/wheat lineages. Since the variability of minisatellites is generally high, such minisatellites can be used to find polymorphisms among the mitochondrial genomes of wild and cultivated barley.

\section{Conclusions}

The mitochondrial genome of barley was similar to those of other grass species in terms of gene content, but the configuration of the genes was quite different from that of wheat, which was used as a reference. Our data show that mitochondrial genome information is essential for correct annotation of the barley nuclear genome. Analysis of our newly generated mitochondrial sequence identified a significant number of fragmented mitochondrial sequences in the nuclear genome, plastid genomes and (importantly) un-anchored genomic sequences. The small polymorphisms in the mitochondrial genome sequences between the wild and cultivated barley lines examined in this study should be further explored to help confirm that the diversity of barley mitochondrial genome sequences is indeed small. Alternatively, we may 
have chosen samples with similar gene pools in terms of mitochondrial variation.

\section{Additional files}

Additional file 1: Table S1. a Primers used for gap filling in the $\mathrm{H} 602$ mitochondrial genome, b Primers used for gap filling in the Haruna Nijo mitochondrial genome. (XLSX $14 \mathrm{~kb}$ )

Additional file 2: Table S2. Primer pairs used for SNP analysis. (XLSX 9 kb) Additional file 3: Table S3. a Contigs of the mitochondrial genome of H602 generated by 454 sequencing, b Contigs of the mitochondrial genome of Haruna Nijo generated by 454 sequencing. (XLSX 13 kb)

Additional file 4: Table S4. Genes identified in the mitochondrial genome of barley. (XLSX $18 \mathrm{~kb}$ )

Additional file 5: Table S5. Novel ORFs in the barley mitochondrial genome. (XLSX $15 \mathrm{~kb})$

Additional file 6: Table S6. Repeated sequences in the mitochondrial genome of H602. (XLSX $15 \mathrm{~kb}$ )

Additional file 7: Table S7. Syntenic region between the mitochondrial genome and chloroplast genome in barley. (XLSX $12 \mathrm{~kb}$ )

Additional file 8: Table S8. BLASTN analysis between the mitochondrial sequences of $\mathrm{H} 602$ and pseudomolecules of seven chromosomes and un-anchored scaffolds and contigs published in Ensembl Plants Hordeum vulgare (Hordeum_vulgare.ASM32608v1.31.dna_sm.genome.fa.gz). (XLSX $59 \mathrm{~kb}$ )

Additional file 9: Table S9. SNP genotypes of 5 wild and 247 cultivated barleys. (XLSX $22 \mathrm{~kb}$ )

Additional file 10: Figure S1. Alignment of protein sequences encoded in the mitochondrial genomes of grass species. (DOC $740 \mathrm{~kb}$ )

\section{Abbreviations}

BAC: Bacterial artificial chromosome; ORF: Open reading frame;

PCR: Polymerase chain reaction; SNP: Single nucleotide polymorphism

\section{Acknowledgments}

We thank Dr. Tsuyoshi Tanaka for repeat sequence analysis and Ms. Yuka Motoi, Ms. Hiromi Ando and Ms. Nami Yamaji for technical assistance. Barley seeds were provided by the National Bioresource Project of Barley, MEXT of Japan.

\section{Funding}

KS is supported by the scientific technique research promotion program for agriculture, forestry fisheries and food industry of Japan (grant Number 25013A) and JSPS KAKENHI (grant Number JP15K07257). TT is supported by JSPS KAKENHI (grant Number JP26292007).

\section{Availability of data and materials}

Sequence data from this article have been submitted to the DDBJ/EMBL/ GenBank Data Libraries under accession numbers AP017300 and AP017301 for H602 and Haruna Nijo, respectively. Phylogenetic data in Fig. 4 are available from Treebase (http://purl.org/phylo/treebase/phylows/study/ TB2:S19756)

\section{Authors' contributions}

$\Pi \mathrm{T}$ and $\mathrm{KS}$ designed the research. MT, HH and $\mathrm{HY}$ conducted experiments. $\mathrm{HH}$, TT and KS wrote the paper. All authors read and approved the final manuscript.

\section{Competing interests}

The authors declare that they have no competing interests.

\section{Consent for publication}

Not applicable.

Ethics approval and consent to participate

Not applicable.

\section{Author details}

${ }^{1}$ Institute of Plant Science and Resources, Okayama University, 2-20-1 Chuo, Kurashiki, Okayama 710-0046, Japan. ${ }^{2}$ Plant Organelle Genomics Research Center and Faculty of Life Sciences, Kyoto Sangyo University, Motoyama, Kamigamo, Kita-ku, Kyoto 603-8555, Japan.

Received: 29 April 2016 Accepted: 12 October 2016

Published online: 24 October 2016

\section{References}

1. Zohary D, Hopf E, Weiss M. Domestication of plants in the old world. 4th ed. Oxford University Press; 2012.

2. Pourkheirandish M, Hensel G, Kilian B, Senthil N, Chen G, Sameri M, Azhaguvel P, Sakuma S, Dhanagond S, Sharma R, et al. Evolution of the grain dispersal system in barley. Cell. 2015;162:527-39.

3. Sato K, Flavell A, Russell J, Börner A, Valkoun J. Biotechnological approaches to barley improvement. In: Kumlehn J, Stein N, editors. Biotechnology in agriculture and forestry. Heidelberg: Springer; 2014. p. 21-36.

4. International Barley Sequencing Consortium (IBSC). A physical, genetic and functional sequence assembly of the barley genome. Nature. 2012;491:711-6.

5. Komatsuda T, Pourkheirandish M, He C, Azhaguvel P, Kanamori H, Perovic D, Stein N, Graner A, Wicker T, Tagiri A, et al. Six-rowed barley originated from a mutation in a homeodomain-leucine zipper I-class homeobox gene. Proc Natl Acad Sci U S A. 2007;104:1424-9.

6. Taketa S, Amano S, Tsujino Y, Sato T, Saisho D, Kakeda K, Nomura M, Suzuki T, Matsumoto T, Sato K, et al. Barley grain with adhering hulls is controlled by an ERF family transcription factor gene regulating a lipid biosynthesis pathway. Proc Natl Acad Sci U S A. 2008;105:4062-7.

7. Ahokas H. Cytoplasmic male sterility in barley: evidence for the involvement of cytokinins in fertility restoration. Proc Natl Acad Sci U S A. 1982;79:7605-8.

8. Kubo T, Newton KJ. Angiosperm mitochondrial genomes and mutations. Mitochondrion. 2008:8:5-14.

9. Tsunewaki K, Matsuoka Y, Yamazaki Y, Ogihara Y. Evolutionary dynamics of wheat mitochondrial gene structure with special remarks on the origin and effects of RNA editing in cereals. Genes Genet Syst. 2008:83:301-20.

10. Ogihara Y, Yamazaki Y, Murai K, Kanno A, Terachi T, Shiina T, Miyashita N, Nasuda S, Nakamura C, Mori N, et al. Structural dynamics of cereal mitochondrial genomes as revealed by complete nucleotide sequencing of the wheat mitochondrial genome. Nucleic Acids Res. 2005;33:6235-50.

11. Cui P, Liu H, Lin Q, Ding F, Zhuo G, Hu S, Liu D, Yang W, Zhan K, Zhang A, et al. A complete mitochondrial genome of wheat (Triticum aestivum $\mathrm{Cv}$. Chinese Yumai), and fast evolving mitochondrial genes in higher plants. J Genet. 2009:88:299-307.

12. Noyszewski AK, Ghavami F, Alnemer LM, Soltani A, Gu YQ, Huo N, Meinhardt S, Kianian PM, Kianian SF. Accelerated evolution of the mitochondrial genome in an alloplasmic line of durum wheat. BMC Genomics. 2014;15:1-16.

13. Middleton CP, Senerchia N, Stein N, Akhunov ED, Keller B, Wicker T, Kilian B. Sequencing of chloroplast genomes from wheat, barley, rye and their relatives provides a detailed insight into the evolution of the Triticeae tribe. PLoS One. 2014;9:e85761.

14. Saisho D, Myoraku E, Kawasaki S, Sato K, Takeda K. Construction and characterization of a Bacterial Artificial Chromosome (BAC) library from the Japanese malting barley variety Haruna Nijo. Breed Sci. 2007;57:29-38.

15. Sato K, Shin-I T, Seki M, Shinozaki K, Yoshida H, Takeda K, Yamazaki Y, Conte M, Kohara Y. Development of 5006 full-length CDNAs in barley: a tool for accessing cereal genomics resources. DNA Res. 2009;16:81-9.

16. Matsumoto T, Tanaka T, Sakai H, Amano N, Kanamori H, Kurita K, Kikuta A, Kamiya $\mathrm{K}$, Yamamoto M, Ikawa $\mathrm{H}$, et al. Comprehensive sequence analysis of 24,783 barley full-length cDNAs derived from 12 clone libraries. Plant Physiol. 2011;156:20-8.

17. Sato K, Tanaka T, Shigenobu S, Motoi Y, Wu J, Itoh T. Improvement of barley genome annotations by deciphering the Haruna Nijo genome. DNA Res. 2016;23:21-8.

18. Sato K, Nankaku N, Takeda K. A high density transcript linkage map of barley derived from a single population. Heredity. 2009;103:110-7.

19. Bonen L, Gray MW. Organization and expression of the mitochondrial genome of plants I. The genes for wheat mitochondrial ribosomal and transfer RNA: evidence for an unusual arrangement. Nucleic Acids Res. 1980;8:319-35. 
20. Don RH, Cox PT, Wainwright BJ, Baker K, Mattick JS. 'Touchdown' PCR to circumvent spurious priming during gene amplification. Nucleic Acids Res, 1991;19:4008.

21. Alverson AJ, Wei X, Rice DW, Stern DB, Barry K, Palmer JD. Insights into the evolution of mitochondrial genome size from complete sequences of Citrullus lanatus and Cucurbita pepo (Cucurbitaceae). Mol Biol Evol. 2010;27:1436-48.

22. Schattner P, Brooks AN, Lowe TM. The tRNAscan-SE, snoscan and snoGPS web servers for the detection of tRNAs and snoRNAs. Nucleic Acids Res. 2005;33:W686-9.

23. Kohany O, Gentles AJ, Hankus L, Jurka J. Annotation, submission and screening of repetitive elements in Repbase: RepbaseSubmitter and Censor. BMC Bioinformatics. 2006;7:1-7.

24. Tamura K, Peterson D, Peterson N, Stecher G, Nei M, Kumar S. MEGA5: molecular evolutionary genetics analysis using maximum likelihood, evolutionary distance, and maximum parsimony methods. Mol Biol Evol. 2011;28:2731-9.

25. Notsu Y, Masood S, Nishikawa T, Kubo N, Akiduki G, Nakazono M, Hirai A, Kadowaki K. The complete sequence of the rice (Oryza sativa L.) mitochondrial genome: frequent DNA sequence acquisition and loss during the evolution of flowering plants. Mol Genet Genomics. 2002;268:434-45.

26. Clifton SW, Minx P, Fauron CM, Gibson M, Allen JO, Sun H, Thompson M, Barbazuk WB, Kanuganti S, Tayloe C, et al. Sequence and comparative analysis of the maize NB mitochondrial genome. Plant Physiol. 2004;136:3486-503.

27. Michalovova M, Vyskot B, Kejnovsky E. Analysis of plastid and mitochondrial DNA insertions in the nucleus (NUPTs and NUMTs) of six plant species: size, relative age and chromosomal localization. Heredity. 2013;111:314-20.

28. Sato K, Takeda K. An application of high-throughput SNP genotyping for barley genome mapping and characterization of recombinant chromosome substitution lines. Theor Appl Genet. 2009;119:613-9.

29. Wicker T, Narechania A, Sabot F, Stein J, Vu GT, Graner A, Ware D, Stein N. Low-pass shotgun sequencing of the barley genome facilitates rapid identification of genes, conserved non-coding sequences and novel repeats, BMC Genomics. 2008;9:1-15.

30. Darling AE, Mau B, Perna NT. progressiveMauve: multiple genome alignment with gene gain, loss and rearrangement. PLoS One. 2010;5:e11147.

31. Huang S, Sirikhachornkit A, Faris JD, Su X, Gill BS, Haselkorn R, Gornicki P. Phylogenetic analysis of the acetyl-CoA carboxylase and 3phosphoglycerate kinase loci in wheat and other grasses. Plant Mol Biol. 2002:48:805-20.

32. Braun H-P, Binder S, Brennicke A, Eubel H, Fernie AR, Finkemeier I, Klodmann J, König A-C, Kühn K, Meyer E, et al. The life of plant mitochondrial complex I. Mitochondrion. 2014;19(Part B):295-313.

33. Raczynska KD, Le Ret M, Rurek M, Bonnard G, Augustyniak H, Gualberto JM. Plant mitochondrial genes can be expressed from mRNAs lacking stop codons. FEBS Lett. 2006;580:5641-6.

34. Lamattina L, Gonzalez D, Gualberto J, Grienenberger J-M. Higher plant mitochondria encode an homologue of the nuclear-encoded 30-kDa subunit of bovine mitochondrial complex I. Eur J Biochem. 1993;217:831-8.

35. Honma Y, Yoshida Y, Terachi T, Toriyama K, Mikami T, Kubo T. Polymorphic minisatellites in the mitochondrial DNAs of Oryza and Brassica. Curr Genet. 2011;57:261-70.

36. Bonen $\mathrm{L}$, Bird $\mathrm{S}$. Sequence analysis of the wheat mitochondrial atp6 gene reveals a fused upstream reading frame and markedly divergent $\mathrm{N}$ termini among plant ATP6 proteins. Gene. 1988;73:47-56.

37. Kubo N, Salomon B, Komatsuda T, Bothmer R, Kadowaki K. Structural and distributional variation of mitochondrial rps 2 genes in the tribe Triticeae (Poaceae). Theor Appl Genet. 2005;110:995-1002.

\section{Submit your next manuscript to BioMed Central and we will help you at every step:}

- We accept pre-submission inquiries

- Our selector tool helps you to find the most relevant journal

- We provide round the clock customer support

- Convenient online submission

- Thorough peer review

- Inclusion in PubMed and all major indexing services

- Maximum visibility for your research

Submit your manuscript at www.biomedcentral.com/submit
Biomed Central 